\title{
Circular dichroism in valence photoelectron spectroscopy of free unoriented chiral molecules: Camphor and bromocamphor
}

\author{
T. Lischke, ${ }^{1,2, *}$ N. Böwering, ${ }^{1, \dagger}$ B. Schmidtke, ${ }^{1}$ N. Müller, ${ }^{1}$ T. Khalil, ${ }^{1}$ and U. Heinzmann ${ }^{1}$ \\ ${ }^{1}$ Fakultät für Physik, Universität Bielefeld, 33501 Bielefeld, Germany \\ ${ }^{2}$ Fritz-Haber-Institut der Max-Phalack-Gesellschaft, Faradayweg 4-6, 14915 Berlin, Germany
}

(Received 17 March 2004; published 19 August 2004)

\begin{abstract}
The circular dichroism in the photoelectron angular distribution was investigated for valence photoionization of randomly oriented pure enantiomers of camphor and bromocamphor molecules using circularly polarized light in the vacuum ultraviolet. The forward-backward electron emission spectra were recorded simultaneously with two spectrometers at several opposite angles relative to the propagation direction of the photon beam and compared for each of the two substances. Measurements were also carried out for reversed light helicity and opposite molecular handedness. For the left- and right-handed enantiomers of both molecules we observed asymmetries of comparable magnitude up to several percent. The measured asymmetry parameters vary strongly for different orbital binding energies and also for the selected photon energies in the valence region. The results for both molecules are compared. They suggest a strong influence of the final states on the asymmetry, depending on the chiral geometry of the molecular electronic structure, as well as a significant dependence on the initial states involved. They also confirm theoretical predictions describing the effect in pure electric-dipole approximation.

DOI: 10.1103/PhysRevA.70.022507

PACS number(s): 33.55.Ad, 33.60.Cv
\end{abstract}

\section{INTRODUCTION}

Chirality is a common phenomenon in nature. Many organic molecules are chiral, that means, they have neither a symmetry plane nor a center of inversion. Both forms (enantiomers) of a chiral molecule are mirror images of each other and cannot be superimposed.

Interactions with chiral molecules exhibit strong enantiomeric selectivity. The asymmetries, which arise from the interaction of polarized light with chiral molecules, were first investigated by Biot [1] and then by Pasteur [2]. This quality of chiral molecules is referred to as optical activity (OA). On the one hand it is the starting point of diverse abiotic theories for understanding the origin of homochirality in nature. On the other hand it is a basis for different spectroscopic methods for the study of chiral molecules. Today, optical techniques, which are based on the circular dichroism (CD) in photoabsorption or optical rotation (OR), are routinely used for investigations in analytic chemistry. Both effects result from an interference between electric and magnetic dipole terms, and their magnitude is naturally very small $(<0.01 \%)$. In consequence, the application of these optical methods is usually limited to samples with high concentration in solutions. The wavelength range employed is restricted to the spectral region from the infrared to the ultraviolet.

In order to extend the studies of chiral molecules interacting with polarized light to processes, such as photoionization, dissociation, or predissociation, photon energies in the vacuum ultraviolet (vuv) energy region are needed. The extension of such investigations to chiral molecules with ion-

\footnotetext{
*Electronic address: lischke@fhi-berlin.mpg.de

†Present address: Cymer Inc., 17075 Thornmint Ct., San Diego, CA 92127, USA.
}

izing radiation is an important requirement for the understanding of the effects of handedness in molecular breakup processes. Using synchrotron radiation it is, in principle, possible to carry out these investigations with circularly polarized light in the necessary higher-energy ranges. Such experiments require high-vacuum conditions. Therefore investigations of the $\mathrm{CD}$ and $\mathrm{OR}$ with ionizing radiation usually entail measurements in the gas phase. However, due to the weakness of these effects an extremely highmeasurement sensitivity is needed [3]. On the other hand, such gas-phase experiments have the advantage that there are no interactions between the solvent and the substance to be investigated.

In analogy to the dichroism in photoabsorption, the lack of symmetry of chiral molecules should also lead to an optical activity in photoemission. Such chirality-dependent effects occur even if the molecules are randomly oriented. However, up to now, only a few studies were performed on this subject.

Like the conventional $\mathrm{CD}$ in photoabsorption, the $\mathrm{CD}$ in photoemission occurs not in electric dipole approximation, but only in higher approximations if it is not detected angle resolved. However, as was pointed out by Ritchie [4] and also discussed in detail by Cherepkov [5,6], in contrast to this effect the circular dichroism in the angular distribution (CDAD) for photoionization of randomly oriented chiral molecules can already appear in pure electric dipole approximation. As a consequence, this CD in the differential photoionization cross section is expected to be significantly larger than the $\mathrm{CD}$ in photoabsorption or the $\mathrm{CD}$ of the total (angleintegrated) photoelectron yield. For the case of oriented molecules, similar polarization dependencies of differential photoionization cross sections were discussed theoretically by Ritchie [7], Cherepkov and Kuznetsov [8], and Dubs et al. [9] and have been found in a number of experimental 
investigations [10-14]. However, for unoriented nonchiral targets, these effects usually cancel out due to averaging by integration over all possible directions of the molecular axes.

Generally the dependence of the photoelectron intensity $I$ on the emission angle $\theta$ of the ejected photoelectron relative to the incident beam direction is given in terms of the cross section $\sigma$, the $\beta$ parameter, and the second Legendre polynomial $P_{2}(\cos \theta)=\frac{3}{2} \cos ^{2} \theta-\frac{1}{2}$. However, for the special case of the photoionization of unoriented chiral molecules with circularly polarized light, an additional contribution arises due to the reduced symmetry. It depends on the first Legendre polynomial $P_{1}(\cos \theta)=\cos \theta$, a coefficient $D$, and the light helicity $m= \pm 1$ ( $\sigma^{+}$and $\sigma^{-}$light, respectively). The photoelectron intensity $I(\theta)$ can then be written as

$$
I(\theta)=\frac{\sigma}{4 \pi}\left[1-\frac{\beta}{2}\left(\frac{3}{2} \cos ^{2} \theta-\frac{1}{2}\right)+m D \cos \theta\right] .
$$

The coefficients $\beta$ and $D$ contain angular coupling terms in combination with photoionization matrix elements. Since the $D$ parameter vanishes for nonchiral systems, it has to change its sign for chiral targets if the handedness of the target molecules is reversed. Thus, the interaction between molecules of one handedness with photons of one helicity results in a forward-backward asymmetry in the angular distribution of the emitted photoelectrons. Thus the intensities of the electrons ejected in the forward and backward directions differ in their dependence on the light helicity. In principle this CDAD arising from the last term of Eq. (1) is experimentally accessible by a reversal of either the molecular chirality, the light helicity, or the emission direction of the photoelectrons.

Although this effect was predicted more than 20 years ago, until recently there was neither a quantitative theoretical estimation about its magnitude nor any experimental verification. A few years ago the first numerical calculations were made by Powis for some representative chiral molecules $[15,16]$. Measurable effects of the order of $10 \%$ for valence shell ionization were predicted, with a strong dependence on the molecular structure and the initial state. At the time of writing, we became aware of another theoretical study on the CDAD of other chiral molecules by Stener et al. [17] pointing out the sensitivity of the effect on changes in the electronic structure. To our knowledge, no CDAD calculations have been carried out for valence ionization of camphor derivatives so far.

An experimental observation of the CDAD in the $16.2 \mathrm{eV}$ valence photoionization of bromocamphor $\left(\mathrm{C}_{10} \mathrm{H}_{15} \mathrm{BrO}\right)$ was reported by our group [18]. In agreement with the analytic dependence of the third term in Eq. (1), an asymmetry in the forward-backward electron emission up to several percent had been measured and found to vary as a function of the binding energy. In addition, general implications of this effect for biomolecules and its importance for the origin of terrestrial homochirality were pointed out. Very recently two other experimental results have also been reported on the CDAD of the prototypical chiral molecule camphor $\left(\mathrm{C}_{10} \mathrm{H}_{16} \mathrm{O}\right)$ by Hergenhahn et al. [19] and Garcia et al. [20]. Hergenhahn et al. observed an asymmetry of the electron emission by inner-shell photoionization of the carbonyl C $1 s$ orbital, which was confirmed by electron multiple-scattering calculations. Garcia et al. investigated the angular distribution from the highest occupied molecular orbital (HOMO) by photoionization of camphor. The size of the observed effects was similar in magnitude to our results for bromocamphor. In all these experimental studies, however, the effect was only probed for a selected initial state or at a single photon energy. This made it difficult to differentiate the dependence of the contributions to the CDAD from the initial and final states. Furthermore, only limited conclusions on the influence of the molecular structure on the CDAD could be made from these experiments.

In this paper we present a more comprehensive analysis comparing experimental measurements of the CDAD in the photoionization of enantiomerically pure gas-phase samples of both camphor and bromocamphor with circularly polarized vuv radiation. The dependence of the effect on the emission from different valence orbitals has been studied at different photon energies. In the entire investigated range of valence states, sizable dichroitic effects of up to several percent were observed for both substances. Predominantly we found strong variations in the dependence of the circular dichroism on the kinetic energy of the emitted photoelectrons and also on the selected photon energy.

\section{TARGET SELECTION AND MOLECULAR STRUCTURE}

At the beginning of our experiments only qualitative estimates existed about the CDAD of free unoriented chiral molecules. There were no predictions on the magnitude of the effect, about the influence of the molecular structure or about a possible correlation between the CDAD and conventional optical activity of the molecules. For our study we set the following conditions to be met by the chosen chiral molecules: (1) In order to be able to work in the gas phase it was necessary to achieve a high-target gas density of the molecules without inducing any thermal dissociation. (2) In order to reduce the complexity of target preparation the substances had to be commercially available in both types of handedness (enantiomers) with high purity. (3) At least two chiral molecules were to be examined in order to obtain information about the influence of the molecular structure. The chiral $D$ parameter for the CDAD of unoriented chiral molecules is proportional to the differences between pairs of dipole matrix elements, which differ from each other by the sign of all the projections of orbital momenta and spins. Although an accurate estimation of these differences is complicated [5], it may be concluded that they should be proportional to a characteristic asymmetry factor $\eta$ discussed by Rich et al. [21]. This factor $\eta$ should depend on the degree of dissymmetry in the structure of the molecule. (4) As was predicted theoretically by Farago [22], and by Fandreyer et al. [23], the spin-orbit (SO) interaction plays an important role in the formation of the electron dichroism (ED) in the electron scattering from chiral molecules. In corresponding experiments for the scattering of spin-polarized electrons from chiral molecules in the gas phase by Mayer and Kessler [24], Nolting et al. [25], and Kessler [26], the scattering 
cross sections showed a spin-related dependence. While no detectable effect was found in the case of camphor consisting only of light elements, for bromocamphor and other chiral molecules containing at least a heavier atom small asymmetries of the order of $10^{-4}$ were observed. Apparently, the ED effect increases strongly with growing nuclear charge $Z$ of the atoms involved in the scattering process, see also [21]. However, for the case of the CDAD in photoionization examined here, there is no such predicted dependence on $Z$ and the SO interaction. For the particular case that the photoelectrons corresponding to different fine-structure components of the final molecular ion state can be separated (provided the initial state is not split), the theoretical work predicts that there are no other small contributions to the asymmetry coefficient $D$. Therefore, the CDAD should depend on the molecular dissymmetry as discussed above, independent of whether the multiplet structure of the ionic state is resolved or not [6]. Correspondingly, no differences between the SO split final states were observed in CDAD measurements for adsorbed achiral $\mathrm{CH}_{3} \mathrm{I}$ molecules aligned on a surface [27]. Nevertheless, for the case of unoriented molecules a possible dependence of the CDAD on the SO interaction may be tested by increasing the nuclear charge $Z$ of the atoms in analogy to the ED effect. Therefore one of the molecules should include at least one atom with $Z>30$.

The chosen chiral molecules are the enantiomers of camphor $[(1 R)-(+)$-camphor, $(1 S)-(-)$-camphor $]$ and the enantiomers of bromocamphor $[([1 R]$-endo)-(+)-3-bromocamphor, ([1S]-endo)-(-)-3-bromocamphor]. The latter are formed when in the camphor molecules a $\mathrm{H}$ atom is substituted by a $\mathrm{Br}$ atom. The geometrical structures of the (1S)-(-)-enantiomers of both molecules are shown at the top of Fig. 1. Also displayed are the calculated molecular orbital surfaces for both molecules for the HOMO, nominally the carbonyl $n_{O}$ orbital, as well as the two next occupied molecular orbitals HOMO-1 and HOMO-2. Calculations for a number of occupied molecular orbitals were made by using the GAUSSIAN 98 package [28]. In order to obtain optimized geometrical parameters, a restricted Hartree-Fock (RHF) calculation was carried out with the help of the STO-3G basis set for camphor and the 6-311G basis set for bromocamphor. For the HOMO of both molecules, the electron density is localized at several atoms in the vicinity of the carbonyl group. In the case of bromocamphor, the next orbitals lead to a localization near the $\mathrm{Br}$ atom. For both molecules, lower occupied molecular orbitals exhibit fairly delocalized electron density spreads over larger molecular bond regions.

The suitability of the selected molecules for experiments in the gas phase was known from previous studies $[18,24,29]$. The chiral samples were acquired with an enantiomeric purity of $98 \%$ (Sigma-Aldrich). It was expected that the similarity of camphor and bromocamphor would be especially useful for examining the dependence of the CDAD on the molecular structure. An additional advantage for such a comparison is the rigid bridged structure of both molecules, which eliminates any uncertainty originating from the occurrences of multiple conformations. Furthermore, in analogy to the ED experiments, a possible spin-dependent effect should be considerably stronger in bromocamphor than in camphor due to the $\mathrm{Br}$ atom with $Z=35$ [24-26].
(1S)-(-)-camphor
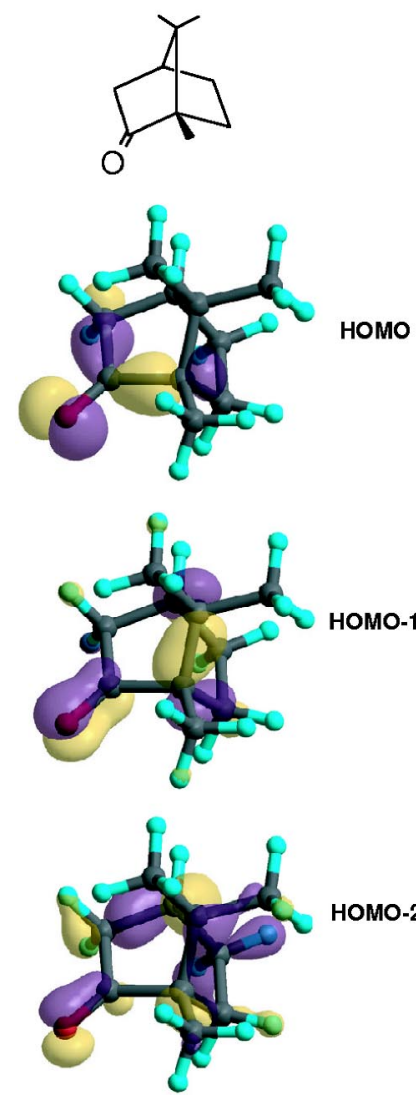

номо
(1S)-(-)-bromocamphor<smiles>CC1(C)[C@H]2CC[C@@H](C2)[C@H]1O</smiles>
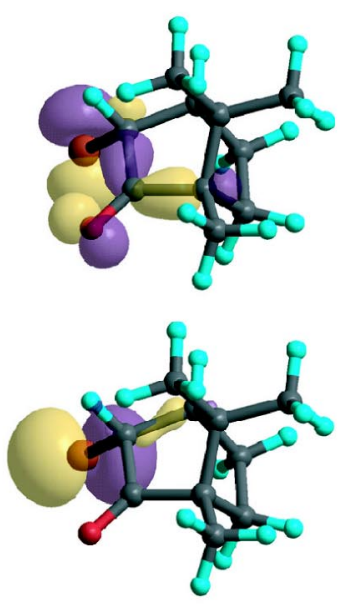

Hомо-2



FIG. 1. (Color online) Geometric and electronic molecular structures of the (1S)-(-) enantiomers of the two target molecules camphor and bromocamphor. Below the respective geometries the RHF-optimized calculated molecular orbital surfaces (RHF/ STO-3G for camphor, RHF/6-311G for bromocamphor) are shown for the HOMOs of the two molecules, nominally the carbonyl $n_{O}$ orbital, as well as the two next occupied molecular orbitals HOMO-1 and HOMO-2. Positive nodes are drawn in light gray (online yellow) and negative nodes are drawn in dark gray (online purple).

\section{EXPERIMENTAL DETAILS AND DATA ANALYSIS}

For the study of the CDAD of unoriented chiral camphor and bromocamphor molecules, an experiment was set up in which the molecules were excited by circularly polarized vuv light and then analyzed energy- and angle-resolved by means of photoelectron spectroscopy. In order to isolate the effects resulting solely from the molecular chirality, the experiments were carried out on molecules in the gas phase where they are naturally unoriented and not aligned. Moreover, apparatus asymmetries had to be eliminated as much as possible by the detection scheme employed.

The experimental setup consisted of three parts: The heated sample source to promote the target substances into the gas phase and to transfer them to the interaction region, the source of circularly polarized VUV light, and the angleresolving electron spectrometer system within a high vacuum chamber. A scheme of our experimental setup is shown in Fig. 2. The enantiomeric $(R)$ and $(S)$ samples of camphor and 




FIG. 2. Scheme of the experimental setup at the $6.5 \mathrm{~m}$ normalincidence monochromator (NIM) at BESSY I. The direction of the incident light and the photoelectron momentum vector $k_{e}$ span the plane of photoelectron collection. $\theta$ is the ejection angle of the photoelectrons relative to the incident beam direction.

bromocamphor consisting of solid powders at room temperature were heated in stainless steel sample containers outside of the vacuum chamber. This was done through controlled heating to $50{ }^{\circ} \mathrm{C}$ in the case of camphor and to $70{ }^{\circ} \mathrm{C}$ in the case of bromocamphor. The resulting vapors were conducted through a heated gas pipe system to the ionization region in the chamber. The gas supply system consisted of valves, a gas pipe, and a nozzle (1 $\mathrm{mm}$ diam). This permitted a fast change from one sample to the other during the measurements. The background pressure within the differentially pumped vacuum chamber was less than $4 \times 10^{-4}$ mbar.

The experiments were carried out at the $6.5 \mathrm{~m}$ normalincidence monochromator (NIM) of the Berlin electron storage ring for synchrotron radiation I (BESSY I) [30]. This monochromator supplied the required circularly polarized light with photon energies up to $24 \mathrm{eV}$. By means of movable horizontal slit apertures producing a variable open gap in front of the monochromator, it was possible to choose the helicity and the degree of the light polarization. As indicated in Fig. 2 the circularly polarized light $\left(\sigma^{+}\right.$and $\sigma^{-}$, respectively) was selected when the slit was above or below the storage ring plane. The monochromator consists of a spherical-mirror-plane-grating arrangement. With the 1200 lines $\mathrm{mm}^{-1}$ grating used here, an intensity of 12 $\times 10^{11}$ photons $s^{-1}$ at $h \nu=20 \mathrm{eV}$ was obtained (for a ring current of $500 \mathrm{~mA}$ ). By adjusting the slits of the monochromator the photon bandwidth was held at $0.2 \mathrm{eV}$, and the degree of circular polarization was set to $P=0.92 \pm 0.03$. Using this value, the measured CDAD asymmetries were normalized to $P=1$.

It is useful to form a quantity independent of the absolute intensity as a measure of the circular dichroism in photoemission. Furthermore, instead of deriving an asymmetry $A_{C D A D}$ from sequential intensity measurements with $\sigma^{+}$and $\sigma$ - light according to the usual method [14], we choose an $A_{C D A D}$ that results from simultaneous measurements at two opposite emission directions (see Fig. 2) in accordance with

$$
A_{C D A D}^{m}=\frac{I^{m}(\theta)-I^{m}\left(\theta+180^{\circ}\right)}{I^{m}(\theta)+I^{m}\left(\theta+180^{\circ}\right)}=\frac{m D \cos \theta}{1-\frac{\beta}{2}\left(\frac{3}{2} \cos ^{2} \theta-\frac{1}{2}\right)} .
$$

By this recording technique with two detectors, the experimental precision is enhanced because only intensity ratios are needed and vapor pressure fluctuations as well as light intensity changes are automatically eliminated. The photoelectrons were simultaneously collected in the observation plane at the polar emission angles of $\theta$ and $\theta+180^{\circ}$ by means of two identically built electron analyzers mounted opposite to each other. Ceramic channeltron amplifiers served as electron detectors [31]. The electron analyzers were hemispherical spectrometers (50 $\mathrm{mm}$ mean radius) with four-element entrance optics as described in [32] having an acceptance angle of about $\pm 7^{\circ}$. In order to achieve high count rates, the spectrometers were used with a pass energy of $20 \mathrm{eV}$ resulting in a resolution of $0.4 \mathrm{eV}$ (electrons and photons). Due to the imaging characteristics of the electron optics employed and the chosen pass energy, electrons with kinetic energies less than $2.0 \mathrm{eV}$ could not be probed. The whole twospectrometer unit could be rotated around a common axis with an orientation of $45^{\circ}$ relative to the storage ring plane and of $90^{\circ}$ relative to the photon beam (see Fig. 2). The polar angle $\theta$ was variable in a range from $+45^{\circ}$ to $+135^{\circ}$. Magnetic fields were compensated to less than $1 \mu \mathrm{T}$ by three Helmholtz coils in addition to one layer of Mu-metal foil on the chamber walls.

Even after careful alignment of the detection system, the two electron spectrometers did not exhibit identical collection angles, exactly the identical transmission characteristics or the identical overall detection efficiency. Thus, it was necessary to make a second measurement in order to determine a chiral asymmetry $A_{C D A D}$ for which the apparatus-related asymmetries are eliminated as much as possible. Several experimental possibilities were to try to achieve this. Since the reversal of the light helicity at the $6.5 \mathrm{~m} \mathrm{NIM}$ by the aperture scanning technique led to small, but noticeable changes in the focal position of the light beam in the ionization region, small changes of the electron-collection geometry were induced. Likewise, through the interchange of the two spectrometers by a rotation of $180^{\circ}$, it was not possible to eliminate all systematic errors completely. Therefore, as the least intrusive method, the handedness of the vapor samples was interchanged by sequential switching between the $(R)$ and $(S)$ enantiomers without any other changes. A combined asymmetry $A_{C D A D}$, which is free of systematic errors, can then be derived, in accordance with

$$
\begin{aligned}
A_{C D A D}^{m} & =\frac{\sqrt{I_{R}^{m}(\theta) I_{S}^{m}\left(\theta+180^{\circ}\right)}-\sqrt{I_{S}^{m}(\theta) I_{R}^{m}\left(\theta+180^{\circ}\right)}}{\sqrt{I_{R}^{m}(\theta) I_{S}^{m}\left(\theta+180^{\circ}\right)}+\sqrt{I_{S}^{m}(\theta) I_{R}^{m}\left(\theta+180^{\circ}\right)}} \\
& =\frac{m D \cos \theta}{1-\frac{\beta}{2}\left(\frac{3}{2} \cos ^{2} \theta-\frac{1}{2}\right)},
\end{aligned}
$$

with $R$ and $S$ representing the two enantiomers. 
The pulses from the channeltron detectors were amplified by counting electronics and accumulated by means of a LABVIEW $^{\text {TM }}$ program, which also served to control and scan the spectrometer supply voltages. The dark counts of the electronics were less 5 counts $\mathrm{s}^{-1}$. Each recorded intensity spectrum was obtained by averaging several energydistribution-curves (EDC) yielding total count rates of up to $N=10^{6}$ counts s $^{-1}$. The statistical random errors of the intensity values were obtained from $\sqrt{N}$. Depending on the photon energy chosen the time to record an individual EDC curve was between 1 and $5 \mathrm{~min}$. The intensity sets $\left[I_{R}^{m}(\theta), I_{R}^{m}(\theta\right.$ $\left.\left.+180^{\circ}\right), I_{S}^{m}(\theta), I_{S}^{m}\left(\theta+180^{\circ}\right)\right]$ needed to determine the asymmetry $A_{C D A D}^{m}$ in Eq. (3) were measured several times for both enantiomers at the photon energies used and subsequently averaged. The random errors of the asymmetries were formed by means of error propagation from the random errors of the corresponding sets of intensity values.

In contrast to an asymmetry formation via reversal of the light helicity, for the experimental procedure described here, longer measurement times were needed. Occasionally, this led to the occurrence of apparatus asymmetries due to longterm or sudden drifts of the electron beam in the storage ring. This resulted in a very small movement of the light focus behind the exit slit of the monochromator, which in turn changed the respective collection volume of the spectrometers leading to changes in the count rates. Measurement cycles with such apparatus contributions were easily identified by direct comparison to previously measured intensity curves or after the evaluation of the asymmetries. Such data were eliminated because a correction proved impractical. A similar problem was noticed with the reproducibility of the vertical position of the electron beam in the storage ring after each electron injection from the synchrotron. Therefore the set of spectra that belonged to an asymmetry had to be measured in a single filling of the storage ring. Another systematical error source is a resulting small racemic admixture due to the necessary frequent interchange of the enantiomers even though the supply lines were evacuated between changes. However, this accumulated racemic portion was found to be less than $2 \%$. This was accomplished by sufficiently long breaks between the changes of the enantiomers corresponding to the decay rate of the target pressure for the evacuated gas pipe system with closed reservoir valve. Nevertheless, the corresponding systematical error was included in the error analysis. This resulted in slightly asymmetrical error bars for the CDAD asymmetry values.

\section{RESULTS}

\section{A. Angular dependence of the CDAD for bromocamphor}

Some data on the dependence of the CDAD on light helicity and polar emission angle were reported for bromocamphor in [18] and for camphor in $[19,20]$. The main interest of the asymmetry measurements presented here lies in the comparison of the dynamic behavior of CDAD of the different chosen chiral molecules in order to study the relation between the CDAD and the molecular structure. However, in addition to the previously discussed CDAD asymmetries at polar emission angles $\theta=55^{\circ}, 90^{\circ}$, and $125^{\circ}$ at a photon en-

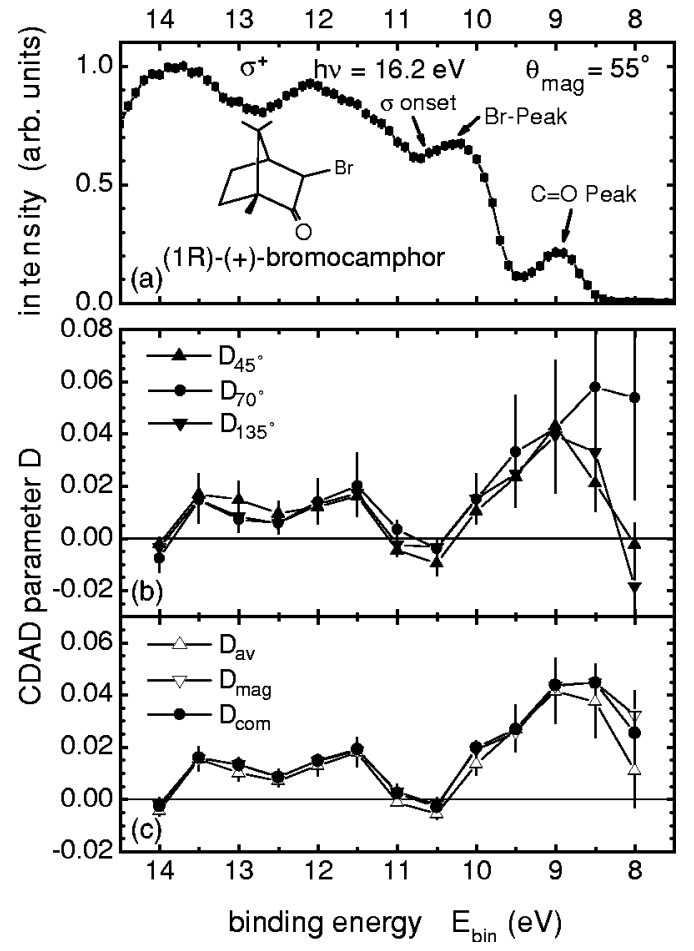

FIG. 3. Circular dichroism in the angular distribution for bromocamphor molecules obtained by photoionization at a photon energy of $16.2 \mathrm{eV}$. (a) Photoelectron intensity spectrum for ([1R]endo)-(+)-3-bromocamphor measured with $\sigma^{+}$light at the magic angle $\theta_{\text {mag }}(\boldsymbol{\square})$. (b) $D$ parameter curves: $D_{45^{\circ}}(\mathbf{\Delta}), D_{70^{\circ}}(\boldsymbol{\bullet})$, and $D_{135^{\circ}}(\boldsymbol{\nabla})$ were obtained from the measured asymmetries $A_{C D A D}(\theta)$ at the corresponding angles with a chosen $\beta=0$. (c) $D_{a v}$ are the averaged $D$ parameters obtained from the three values in (b). For comparison the $D_{\text {mag }}$ data $(\nabla)$ shown in [18] are also plotted. $D_{\text {com }}$ (O) is the averaged curve from all emission angles including the data of [18]. The lines connecting the data points are shown for guidance only.

ergy of $h \nu=16.2 \mathrm{eV}$ [18], further measurements at other emission angles were also performed for bromocamphor to serve as an additional experimental test of the consistency of the analytical description of the angular distribution.

The data measured at $h \nu=16.2 \mathrm{eV}$ are displayed in Fig. 3. A typical photoemission spectrum for ([1R]-endo)(+)-3-bromocamphor as a function of the binding energy $E_{\text {bin }}$ is shown in Fig. 3(a). This intensity spectrum was obtained with $\sigma^{+}$light by averaging several EDC scans recorded at the so-called magic angle $\theta_{\text {mag }}=55^{\circ}$. The scans were taken with binding energy steps $\Delta E_{b i n}=0.1 \mathrm{eV}$ using a spectrometer pass energy of $E_{\text {pass }}=20 \mathrm{eV}$ with a respective combined resolution of $\Delta E=0.4 \mathrm{eV}$. At the magic angle, the second Legendre polynomial vanishes. Consequently, there is no dependence of the intensity on the $\beta$ parameter. Solely the dependencies on the cross section, the light helicity, and the chiral parameter $D$ remain, see Eq. (1).

The first energy band in the spectrum with a binding energy of $9.0 \mathrm{eV}$ results from the electron emission of the nonbinding oxygen orbital $\left(n_{o}\right)$ which is mainly localized near the $\mathrm{C}=\mathrm{O}$ double bond [see Fig. 1(b)]. This molecular orbital represents the most weakly bound electron state in 
many camphor derivatives. A comparison with previous photoelectron studies by Novak et al. [33], Novak and Kovac [34], and Pollmann et al. [35] for camphor and bromocamphor shows that the shoulder at binding energies of $10-11 \mathrm{eV}$ is related to the electron emissions from the lonepair orbitals localized near the $\mathrm{Br}$ atom. The characteristic splitting of $0.31 \mathrm{eV}$ of the two resulting final states due to the spin-orbit coupling of the $\mathrm{Br}$ atom could not be resolved with the overall resolution used in this experiment. The intensity features at higher energies result from emissions of various $\sigma$ bonds within the molecules.

Here, the CDAD for the photoionization of bromocamphor at a photon energy of $h \nu=16.2 \mathrm{eV}$ was examined at the angles $\theta=45^{\circ}, 70^{\circ}, 135^{\circ}$. Samples were taken in binding energy steps $\Delta E_{b i n}=0.5 \mathrm{eV}$. The measured CDAD asymmetries changed the sign when the light helicity was reversed, as expected, and in agreement with our previous results [18]. Combined asymmetries were formed according to

$$
A_{C D A D}(\theta)=\frac{1}{2}\left[A_{C D A D}^{+}(\theta)-A_{C D A D}^{-}(\theta)\right] .
$$

Using Eq. (3) and the energy-dependent angular distribution parameter $\beta$, the chiral parameter $D$ can in principle be determined from any angular measurement according to

$$
D=A_{C D A D} \frac{\left[1-\frac{\beta}{2} P_{2}(\cos \theta)\right]}{\cos \theta} .
$$

However, the values of $\beta$ are not known for bromocamphor, and our experiment was not set up for their explicit measurement. On the other hand, in the angular region investigated, the helicity-independent term $\left[1-(\beta / 2) P_{2}(\cos \theta)\right](\cos \theta)^{-1}$ in Eq. (5) is not strongly dependent on the $\beta$ parameter and does not change its value substantially when $\beta$ changes within the range of $-0.5 \leqslant \beta \leqslant 1.2$. In principle, the possible range for $\beta$ is $-1 \leqslant \beta \leqslant 2$. However, $\beta$ values near the extrema are not to be expected for this large molecule. Angleresolved measurements of the valence photoelectron emission from other organic molecules do not show $\beta$ values of large magnitude [36-38]. Furthermore, the theoretical studies for the other chiral molecules indicate correspondingly small values of $\beta$ [15-17]. In addition, due to the overlapping contributions from many orbitals of bromocamphor with potentially different angular distributions for the region above the $\sigma$ onset, the measured $\beta$ values are not expected to lie near -1 or 2 . Therefore, as an approximation, we have chosen $\beta=0$ in order to carry out the calculation of $D$ from the combined asymmetry curves. However, the possible variation of $\left[1-(\beta / 2) P_{2}(\cos \theta)\right]$ and $D(\beta, \theta)$, respectively, as a function of $\beta$ was included in the error analysis for each particular angle.

Figure 3(b) shows the resulting $D$ parameter versus binding energy for the investigated angles with the sign referring to the $[1 \mathrm{R}]-(+)$ isomer of bromocamphor. Apart from the two points at very low-binding energy (where the counting statistics is poor), there is very good agreement for the $D$ values obtained at different angles. In Fig. 3(c) we plot the averaged data of Fig. 3(b) in comparison with the averaged $D$ parameter obtained from the previous asymmetry results at the two magic angles $\theta_{\text {mag }}=55^{\circ}$ and $\theta_{\text {mag }}=125^{\circ}$ [18]. At these angles $\cos \theta= \pm 1 / \sqrt{3}$; therefore the asymmetry and the $D$ parameter are directly related, independent of the $\beta$ parameter, according to $D=\sqrt{3} A_{C D A D}\left(\theta_{\text {mag }}\right)$. In addition, the combined averaged curve $D_{\text {com }}$ from all emission angles including the data in [18] is also shown for comparison.

To within experimental error the $D$ parameters determined here are in agreement with the values measured directly at the magic angles. This is a further confirmation of the analytical form of the $\cos \theta$ angular dependence of the circular dichroism. It is also an indication that the approximation of $\beta=0$ leads to reasonable results in the case examined here and illustrates that the dichroism is only weakly dependent on the angular-distribution parameter $\beta$ in the region investigated.

In principle, by measuring the $D$ parameter at a magic angle and by an asymmetry measurement at another angle $\left(\theta \neq 90^{\circ}\right)$ the $\beta$ value may be calculated using Eq. (3). However, as mentioned above, this leads to relatively large inaccuracies resulting from the term $1-D / A_{C D A D} \cos \theta$, unless the experimental error of the measured quantities is very small. Nevertheless, this calculation was carried out for the outermost $n_{O}$ orbital at $E_{b i n}=9.0 \mathrm{eV}$, which is the only orbital that could be completely resolved and separated from the other valence orbitals. By averaging all measured asymmetries, $\beta=-0.46 \pm 0.50$ was obtained. This value is comparable to corresponding $\beta$ values that have been found in photoemission measurements at similar photon energies for the outer $n_{O}$ orbital of the $\mathrm{CO}_{2}$ molecule [39].

\section{B. Energy dependence of the CDAD for camphor and bromocamphor}

The photon energy dependence of the CDAD of camphor and bromocamphor was studied for photon energies from $h \nu=13.2 \mathrm{eV}$ to $h \nu=24.2 \mathrm{eV}$. All measurements were carried out at the magic angles in order to accumulate the necessary high total count rates for sufficiently accurate statistics, without any further examinations for the angular dependence of the CDAD. As described above, for measurements at the magic angle the CDAD asymmetry $A_{C D A D}$ is directly related to the $D$ parameter. The samples were taken at binding energy steps $\Delta E_{b i n}=0.25 \mathrm{eV}$ or $\Delta E_{b i n}=0.5 \mathrm{eV}$. The photoemission spectra were measured in the way described in Sec. IV A. Subsequently, the corresponding CDAD asymmetries were evaluated.

The dependence on binding energy of the resulting asymmetries $A_{C D A D}^{+}$and $A_{C D A D}^{-}$for $\sigma^{+}$light and $\sigma^{-}$light are shown for bromocamphor in Figs. 4(b)-4(h) and for camphor in Figs. 5(b)-5(m), respectively. The corresponding $D$ parameter obtained by averaging both asymmetries is also plotted. [Again, the sign of $D$ refers to the $(1 \mathrm{R})-(+)$ enantiomer in each case.] In the case of the asymmetry and the $D$ parameter of bromocamphor at the photon energy of $h \nu=16.2 \mathrm{eV}$ [Fig. 4(d)], the averaged data from all emission angles, including the data of [18], are shown (see Sec. IV A). The measured intensity spectra for camphor and bromocamphor were found to be fairly similar, except for the additional shoulder due to the $n_{B r}$ orbital near $10.5 \mathrm{eV}$ in the case of bromocamphor. For brevity, we display at the top of each figure only the 


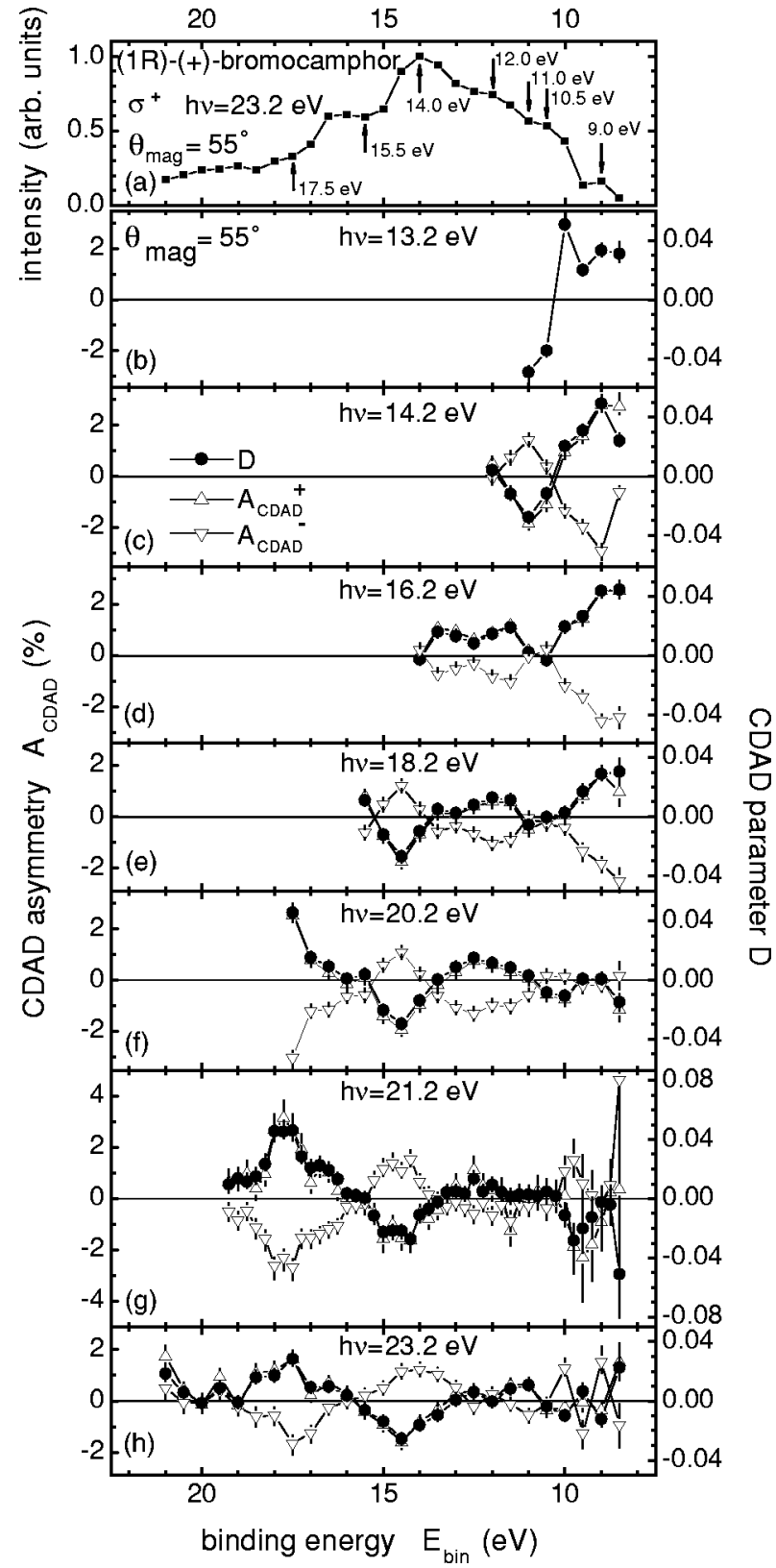

FIG. 4. Energy dependence of the CDAD for bromocamphor obtained for different photon energies at the magic angle $\theta_{\text {mag }}$ $=55^{\circ}$. (a) Intensity spectrum recorded at $h \nu=23.2 \mathrm{eV}$ (ם). The arrows mark the binding energies chosen for the CIS asymmetry plots in Figs. 6(b)-6(h) CDAD asymmetry curves for $\sigma^{+}$light $(\triangle)$ and $\sigma^{-}$ light $(\nabla)$ and the corresponding $D$ parameter determined from both asymmetry values $(\bigcirc)$. The $D$ parameters mask partly the asymmetries of $\sigma^{+}$. The lines connecting the data points are shown for guidance only.

intensity spectrum obtained from the $(R)$ enantiomer of the molecule with $\sigma^{+}$light at the respective highest photon energy [Figs. 4(a) and 5(a), respectively]. Here, samples were taken in binding energy steps $\Delta E_{b i n}=0.1 \mathrm{eV}$ at a pass energy $E_{\text {pass }}=20 \mathrm{eV}$.

The $n_{O}$ band-ionization energies $E_{\text {bin }}\left(n_{O}\right)$ of the camphor derivatives change due to changes of the orbital energies induced by the presence of substituents, like the $\mathrm{Br}$ atoms in the case studied here [33-35]. This results in an energy shift $\Delta E=0.05 \mathrm{eV}$ of the carbonyl peak between camphor $\left[E_{b i n}\left(n_{O}\right)=8.95 \mathrm{eV}\right]$ and bromocamphor $\left[E_{\text {bin }}\left(n_{O}\right)=9.0 \mathrm{eV}\right]$.

In the entire examined valence region for both bromocamphor and camphor molecules, the CDAD effect depends strongly on the binding energy. Moreover, several sign changes are observed as $E_{b i n}$ varies. Within the margins of error, the asymmetries show the predicted symmetrical mirror behavior upon reversal of the light helicity. The resulting $D$ parameters are of comparable magnitude for both substances.

Typically, for all photon energies examined, the value of $D$ is largest in magnitude at binding energies near the $n_{O}$ orbital. Here, the largest differences occur in the CDAD between the two molecules. For bromocamphor, $D\left(n_{O}\right)$ is largest at $h \nu=14.2 \mathrm{eV}$ with a value of $D\left(n_{O}\right)_{\max }=0.049 \pm 0.006$, for camphor at $h \nu=20.2 \mathrm{eV}$ with $D\left(n_{O}\right)_{\max }=-0.078 \pm 0.005$. For the outermost weakly bound states, strong variations of $D$ are observed. This indicates the strong influence of the final states on the CDAD in this binding energy region. Furthermore, for the asymmetries at higher binding energies, sizable differences between both target molecules have been found. At energies in the middle of the examined valence region at $E_{b i n}>10.95 \mathrm{eV}$ for camphor and $E_{b i n}>11.0 \mathrm{eV}$ for bromocamphor, respectively, the asymmetry behavior is fairly similar for both molecules and nearly independent of the photon energy. Here, the influence of the final states on the dichroism seems to be weaker. At photon energies of $h \nu>21.2 \mathrm{eV}$, a reduction of the dichroic effects is observed for the more strongly bound molecular orbitals of camphor $\left(E_{\text {bin }}>17 \mathrm{eV}\right)$.

Additional information about the influence of the final states on the CDAD was obtained from asymmetry curves originating from constant-initial-state (CIS) asymmetry curves. Even though CIS spectra were not recorded directly in our measurements, it is nevertheless possible to form CIS asymmetry curves at a given binding energy from all our measured EDC asymmetries. Because the asymmetries are independent of the intensity, the EDC asymmetry curves can be used directly to determine CIS asymmetry curves. In contrast to this procedure, the corresponding CIS intensity spectra are dependent on the respective normalization and thus more difficult to form. Therefore, only the CIS asymmetry curves are shown here.

We have chosen a set of seven different binding energies for such a comparative study of both molecules. These energies are marked by arrows in the intensity spectra of Figs. 4(a) and 5(a). The CIS asymmetries determined in this way are shown in Figs. 6(a)-6(g). The CDAD parameter values for camphor and bromocamphor are plotted in direct comparison as a function of kinetic electron energy. The chosen binding energies are listed in the figures.

For the HOMO $\left[E_{b i n}=8.95 \mathrm{eV}\right.$, see Fig. 6(a)], the orbital with strong $n_{O}$ character, both substances exhibit a pronounced influence of the dichroism on the final states at the kinetic energies studied. Here the largest differences $\Delta D$ are measured between the smallest and largest $D$ value in the examined valence region. For camphor $\Delta D=0.081$ $\pm 0.008 \mathrm{eV}$ and for bromocamphor $\Delta D=0.062 \pm 0.007 \mathrm{eV}$ is 

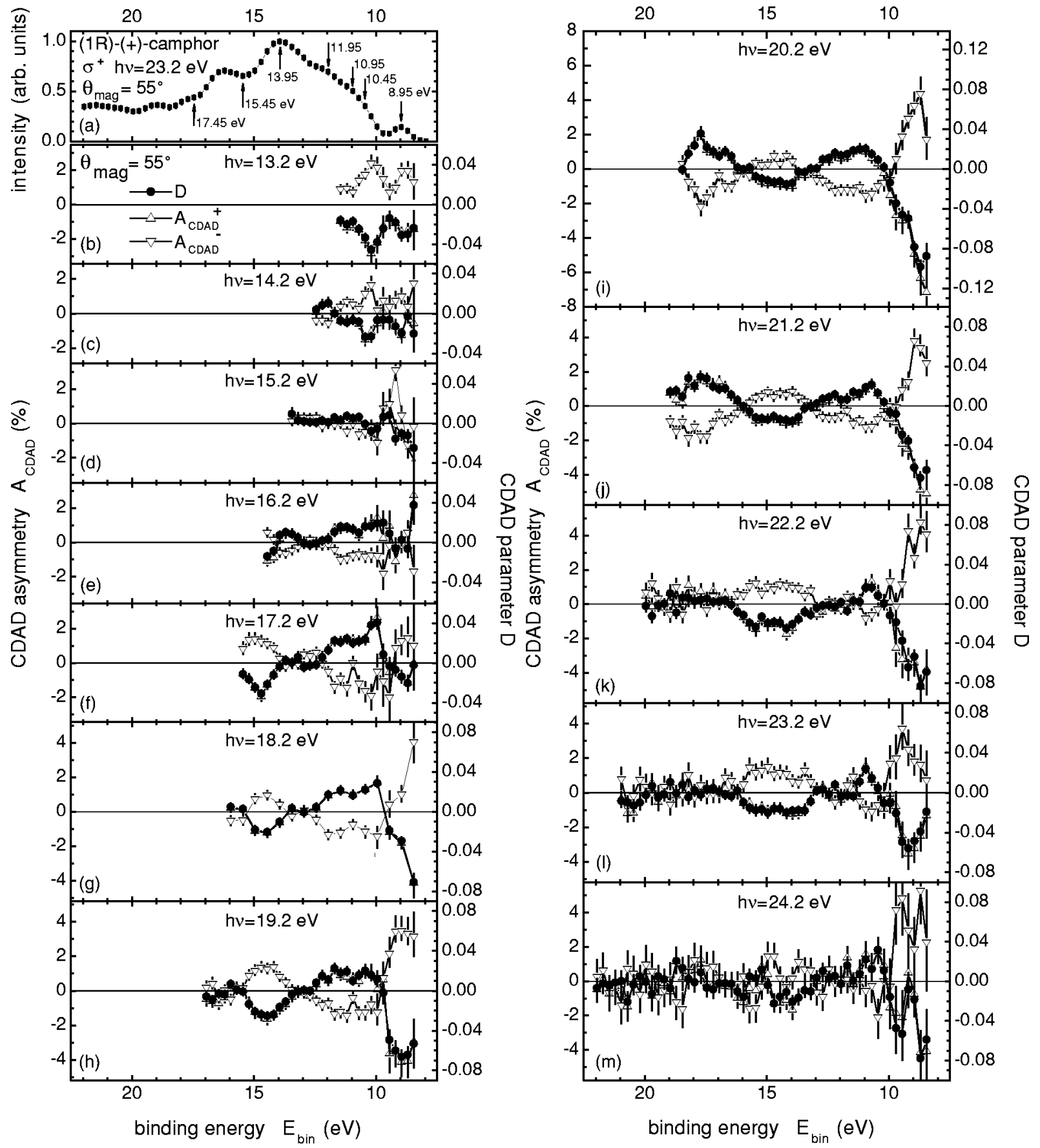

FIG. 5. Energy dependence of the CDAD for camphor obtained for different photon energies at the magic angle $\theta_{\text {mag }}=55^{\circ}$. (a) Intensity spectrum recorded at $h \nu=24.2 \mathrm{eV}(\boldsymbol{\square})$. The arrows mark the binding energies chosen for the CIS asymmetry plots in Figs. 6(b)-6(h) CDAD asymmetry curves for $\sigma^{+}$light $(\triangle)$ and $\sigma^{-}$light $(\nabla)$ and the corresponding $D$ parameter determined from both asymmetry values $(\bullet)$. The $D$ parameters mask partly the asymmetries of $\sigma^{+}$. The lines connecting the data points are shown for guidance only.

obtained. The $D$ parameters of bromocamphor exhibit a distinct trend toward more positive values in comparison to the corresponding data of camphor.

There is a strong change of the asymmetry curves in the next plot $\left[E_{b i n}=10.45 \mathrm{eV}\right.$, see Fig. 6(b)], in comparison to the asymmetries found at the binding energy of the HOMO orbital. For HOMO-1 of bromocamphor, the orbital with strong $n_{B r}$ character [34,35], a sizable decrease of the CDAD is observed at all kinetic energies investigated. Here the di- chroism exhibits a negative trend and is restricted to electrons with small kinetic energies. In the case of camphor the asymmetries at this binding energy already result from emissions of the $\sigma$ onset because there are no orbitals associated with $\mathrm{Br}$. (The $\sigma$ onset for camphor is at $E_{b i n}=10.40 \mathrm{eV}$ [33] and for bromocamphor at $E_{b i n}=10.67 \mathrm{eV}$ [34].)

For binding energies distinctly above the $\sigma$ onset $\left[E_{b i n}\right.$ $\geqslant 10.95 \mathrm{eV}$ see Figs. $6(\mathrm{c})-6(\mathrm{~g})]$, the variations of the $D$ parameter are mainly limited to the continuum states at low 
kinetic electron energies. On the other hand, for larger kinetic energies of the electrons the observed asymmetry curves are predominantly flat. Here the CDAD has only a weak dependence on the continuum states. This is also manifested in a substantial decrease of $\Delta D$. Apparently, for fast electrons the magnitude of the effect is more strongly influenced by the initial state. For all shown CIS spectra, in particular for binding energies above the $\sigma$ onset, there is a large similarity of the asymmetry curves between camphor and bromocamphor. This characteristic similarity is also found in the EDC asymmetries at identical binding energies. The substituted $\mathrm{Br}$ atom seems to induce predominantly a contribution to the dichroism, which is relatively independent of the kinetic energy.

In analogy to the determination of the CIS asymmetry curves shown above, we have also obtained the corresponding constant final state (CFS) asymmetry curves. For the most part, a very similar dependence on photon energy was found for the two molecules. However, not much more additional information could be obtained from these curves. They are, therefore, not plotted here. The close resemblance of the CFS asymmetry curves suggests that the delocalized molecular orbitals of both molecules are fairly similar. This conclusion is also in agreement with the results of the GAUSSIAN 98/RHF molecular orbital calculations (see Sec. II). Vice versa, this shows that for a corresponding final state the similarity of the molecular electronic structure leads also to a similarity of the CDAD asymmetry effect.

\section{DISCUSSION}

The analysis of the explicit expressions of the chiral parameter $D$ derived in the theory reveals that the interference of the outgoing partial waves of adjacent $l$ values gives rise to the CDAD for unoriented chiral molecules [4-6]. Therefore, this CDAD can manifest itself as a final-state effect depending on the chiral geometry that can arise independent of the character of the initial state involved. On the one hand, we observed a strong dependence of the CDAD on the involved final states especially for outgoing electrons with low kinetic energies. On the other hand, however, our measurements give evidence for a significant dependence of the CDAD on the initial states involved for given final states.

In the entire examined valence region we observed CDAD effects for both target molecules (see Figs. 4 and 5). In fact, an asymmetry appears for all initial states involved. In particular there is no direct correlation between the localization of the electron density from the molecular orbital involved and the appearance of a CDAD. For both substances, sizable asymmetries are observed both for emissions from strongly delocalized $\sigma$ orbitals and from the HOMOs that consist mainly of the strongly localized orbital of the carbonyl double bond (see Fig. 1). Furthermore, the electron emissions in bromocamphor originating from the localized lone-pair $n_{\mathrm{Br}}$ orbital with electron density predominantly at the bromium atom and the adjacent carbon atom exhibit a CDAD at low kinetic energies [Fig. 6(b)].

A similar effect was observed for the CDAD in the photoionization of the carbon $\mathrm{C} 1 s$ core orbital from camphor reported in [19]. Here, the initial level has well-defined parity and is strongly localized and isolated from the chiral molecular potential. Thus, both our and these results obtained for localized states demonstrate that a chirality of the involved initial state is not necessarily of strong influence for the occurrence of the CDAD. Rather, these two experimental results lead to the conclusion that the observed CDAD seems to be connected with the sensitivity of the outgoing photoelectron wave function to the characteristic handedness of the molecular potential.

The observed clear differences of the asymmetries from camphor and bromocamphor at low binding energies can be attributed to the different molecular structure of both molecules. The substitution of the $\mathrm{Br}$ atom produces additional contributions in the electron density of the molecular orbitals of bromocamphor localized near the $\mathrm{Br}$ atom. This leads to differences in the electron density of the outermost electron orbitals for these two molecules. This view is confirmed by the molecular orbital calculations for the valence orbitals of both molecules given in Sec. II (Fig. 1). Compared to camphor apparently the addition of electron density into the HOMO near the $\mathrm{Br}$ atom for bromocamphor causes a strong additional positive contribution to the CDAD resulting from electron emissions from this orbital [Fig. 6(a)].

In contrast to these observations for the $n_{O}$ orbital, the CDAD of bromocamphor resulting from electron emissions from the lone-pair orbital near the bromium atom exhibits a strongly negative trend. In comparison, the CDAD of camphor rises to more positive values in this binding energy region [see Fig. 6(b)].

As shown in Fig. 6(c), above the $\sigma$ onset (see Sec. IV B), a different behavior in the asymmetries of camphor and bromocamphor is observed, as well. This provides evidence that for bromocamphor $n_{\mathrm{Br}}-\sigma$ interactions exist in this valence region that sizably influence the CDAD. In corresponding intensity studies of camphor derivatives only for dibromocamphor a significant $n_{\mathrm{Br}}-\sigma$ interaction was seen due to a sizable modification of the SO splitting [34]. In the literature this value is associated with the undisturbed case without additional interactions $[34,40]$. Thus the asymmetry measurement is clearly more sensitive to this interaction than a pure intensity measurement. Apparently the $D$ parameter is strongly influenced by changes of the molecular electronic structure.

With increasing delocalization of the orbitals, the additional electron density distribution in the vicinity of the $\mathrm{Br}$ atom has less influence and the orbitals of camphor and bromocamphor become more similar. Therefore the asymmetry curves of camphor and bromocamphor resemble each other at higher binding energies, especially for emissions at energies in the middle of the examined valence region. This can be seen from the comparison of Figs. 4-6. In our measurements we do not observe an increase of the CDAD for the delocalized orbitals with higher binding energies due to a possibly larger sensitivity to the CDAD effect as was predicted for a different chiral molecule (L-alanine) in [16]. Rather, the $D$ values in this region we found to be normally smaller compared to the $D$ values for the higher occupied orbitals. The reason for this could be that the CDAD from adjacent delocalized electron orbitals, with different CDAD 


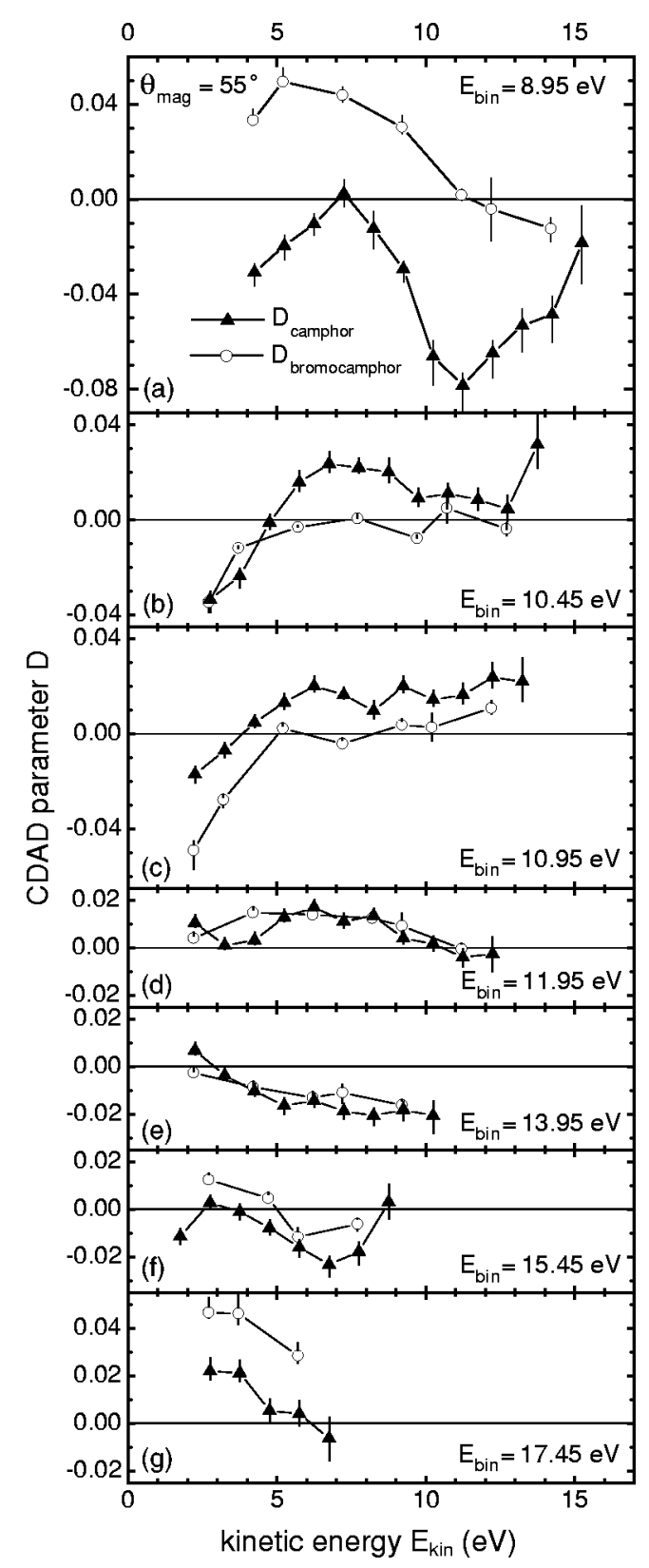

FIG. 6. CIS asymmetry plots for camphor $(\mathbf{\Delta})$ and bromocamphor $(\bigcirc)$ derived from the $D$ parameters for the chosen binding energies in Figs. 4(a) and 5(a), respectively. The binding energies for camphor are listed in the plots. For the corresponding binding energies of bromocamphor a shift of $\Delta E=0.05 \mathrm{eV}$ has been added. The lines connecting the data points are shown for guidance only.

in sign and magnitude, is canceled out due to the limited experimental resolution in our experiment. Calculations for oxirane [17] show similarly that in the case of two combined energy bands, the magnitude of the resulting $D$ parameter is sizable smaller compared to its value for the separated energy bands. On the other hand, the delocalization of the initial states and the resulting additional scattering of the outgoing electron waves at the molecular skeleton could possibly cause additional partial waves leading to more complexly structured interference patterns. This could also result in a decreasing of the CDAD effect. Especially, if the effect is averaged over nonoriented molecules, as in our experiment.

Although a possible influence of the SO interaction on the CDAD could not be directly studied due to the unresolved SO splitting, we can conclude that SO interaction is not necessary for the occurrence of the CDAD because we have measured asymmetries of same magnitude for both substances. Our measurements confirm the theoretical predictions $[5,6]$. It can be surmised that other chiral target molecules consisting only of light atoms with vanishingly small SO interaction may also exhibit a CDAD of a magnitude similar to camphor.

Generally the CDAD is observed to be significantly dependent on the initial states in the examined energy region. On the one hand, this follows from the sign reversals in the EDC asymmetry curves as a function of binding energy (see Figs. 4 and 5). On the other hand, the CDAD depends in fact sensitively on the low energy continuum states $\left(E_{k i n}\right.$ $\leqslant 6 \mathrm{eV}$ ). This can be seen from the large and strongly varying values of the slow outgoing electrons in the CIS asymmetries in combination with the selectivity of the initial states (Fig. 6). However, for faster photoelectrons the CIS asymmetry curve becomes more flat and the CDAD reaches relatively constant values or even disappears. Thus the effect is not selective for energetic higher continuum states. Rather here the magnitude and the sign of the circular dichroism is strongly dependent on the initial state involved. This can also be seen from the similarity of the EDC asymmetry curves for photon energies with $h \nu>16.2 \mathrm{eV}$ [Figs. 4(e)-4(h) and $5(\mathrm{~g})-5(\mathrm{~m})$, respectively].

The flatter shape of the asymmetry curves and the approach of a constant asymmetry value, respectively, above electron energies $E_{k i n} \geq 8 \mathrm{eV}$ (see Fig. 6) compares qualitatively with CDAD valence calculations for other chiral molecules reported in [15-17], in which the asymmetry curves also become flat for $E_{k i n}>8 \mathrm{eV}$. In these calculations, there is also a general trend for $D$ to decrease toward zero as the kinetic energy rises to $\sim 20 \mathrm{eV}$. This gives evidence of a decreasing sensitivity to scattering by the chiral molecular ion cores as the electron energy is increased. A possible explanation for this trend could be that the interference effects between the direct outgoing partial waves and the partial waves backscattered by the chiral molecular potential become smaller with decreasing wavelength of the emitted electron. Due to the directional averaging over the molecular axis orientations sharp interference structures can cancel each other. This could result in a faster decrease of the effect, in contrast to the CDAD of oriented molecules. Furthermore the scattering of fast outgoing electrons could be more strongly influenced by partial waves with higher angular momenta $l$. This leads to more complexly structured interference patterns and could also amplify the decrease of the asymmetries.

Our studies show that already free noninteracting unoriented molecules exhibit sizable dichroitic effects in the vuv region. Thus angle-resolved photoelectron spectroscopy of free unoriented chiral molecules with circularly polarized light is a fundamental experiment in noncoincidence, spinunresolved photoionization.

The observed magnitude up to $6 \%$ and the agreement of the measured CDAD in the angular and helicity dependence 
together with existing theoretical predictions [15-17] as well as the previous experimental results [18-20] show that the CDAD is caused predominantly through the interaction of the chiral molecules with the electric dipole part of the electromagnetic field. In contrast, higher-order terms seem to be insignificant at the present level of accuracy.

Because of the lack of a mirror symmetry the dichroitic effects do not cancel when all molecular axis directions are averaged. This is reflected in the forward-backward intensity asymmetry of the emitted photoelectrons, which also results in an asymmetric momentum distribution of the recoil ions [4]. Therefore the contribution of the momentum transfer to the ions reverses its sign when the handedness of the molecule is changed. Thus even though this CDAD is not an integral effect, it can lead to an enantiomeric separation of chiral molecules by photoionization for circularly polarized light incident from one direction.

\section{CONCLUSIONS}

In this paper we reported comparative studies of the dynamical behavior of the circular dichroism in the photoelectron angular distribution. The experiments were performed on the chiral molecules camphor and bromocamphor in the gas phase using valence photoionization with synchrotron radiation in the vuv range.

Our measurements show that a significant forwardbackward asymmetry of similar magnitude exists for both target molecules in the photoelectron angular distribution for the entire valence region examined. On the one hand, the effect occurs independent of the degree of the localization of the selected initial state. The scattering of the outgoing electron waves at the chiral molecular potential is an intrinsic reason for this kind of CDAD that also results in a strong dependence of the effect on the molecular structure. This can be seen in particular in the strong final-state influence of the asymmetries for the emissions from the more weakly bound electron orbitals. On the other hand, we also observed in our measurements a significant dependence of the CDAD on the initial states involved.

Only comparisons with general trends in theoretical results for the CDAD of other types of unoriented chiral molecules could be made here. It would be of great interest to compare our experimental results with calculations of the dynamical photoionization parameters including dichroism for the valence ionization region of camphor and bromocamphor molecules.

The observed handedness of the CDAD upon reversal of the light helicity, its $\cos \theta$ dependence, and its relatively large magnitude of several percent appearing in our experiments confirm that the effect can be described by a nonrelativistic model in electric dipole approximation. This is in contrast to the conventional $\mathrm{CD}$ in photoabsorption. Our studies give further experimental evidence that the CDAD from unoriented chiral molecules is a general phenomenon. This leads us to the expectation that the effect should also occur with similar magnitude for other chiral molecules even when they consist only of light elements like camphor.

\section{ACKNOWLEDGMENTS}

We are grateful to N. A. Cherepkov for his initiating theoretical predictions and many illuminating discussions. We thank O. Kugeler for help in carrying out some of the molecular orbital calculations. We also thank J. Kessler, G. F. Hanne, K. Blum, U. Hergenhahn, and I. Powis for helpful discussions. Funding by the Deutsche Forschungsgemeinschaft is gratefully acknowledged.
[1] J. B. Biot, Traité de Physique Expérimentale et Mathématique (Deterville, Paris, 1816).

[2] L. Pasteur, Ann. Chim. Phys. III 24, 442 (1848).

[3] T. Müller, K. B. Wiberg, P. H. Vaccaro, J. R. Cheeseman, and M. J. Frisch, J. Opt. Soc. Am. B 13, 125 (2002).

[4] B. Ritchie, Phys. Rev. A 13, 1411 (1976); 14, 359 (1976).

[5] N. A. Cherepkov, Chem. Phys. Lett. 87, 344 (1982).

[6] N. A. Cherepkov, Adv. At. Mol. Phys. 19, 395 (1982); J. Phys. B 16, 1543 (1983).

[7] B. Ritchie, Phys. Rev. A 12, 567 (1975).

[8] N. A. Cherepkov and V. V. Kuznetsov, Z. Phys. D: At., Mol. Clusters 7, 271 (1987).

[9] R. Dubs, S. N. Dixit, and V. McKoy, Phys. Rev. Lett. 54, 1249 (1985).

[10] F. Heiser, O. Geßner, J. Viefhaus, K. Wieliczek, R. Hentges, and U. Becker, Phys. Rev. Lett. 79, 2435 (1997).

[11] C. Westphal, J. Bansmann, M. Getzlaff, and G. Schönhense, Phys. Rev. Lett. 63, 151 (1989).

[12] J. Bansmann, C. Ostertag, G. Schönhense, F. Flegel, C. Westphal, M. Getzlaff, F. Schäfers, and H. Petersen, Phys. Rev. B 46, 151 (1992).

[13] G. Schönhense, Phys. Scr. T31, 255 (1990).
[14] G. Schönhense and J. Hormes, in VUV and Soft X-Ray Photoionisation, edited by U. Becker, and D. A. Shirley (Plenum, New York, 1996), p. 607.

[15] I. Powis, J. Chem. Phys. 112, 301 (2000).

[16] I. Powis, J. Chem. Phys. 104, 878 (2000).

[17] M. Stener, G. Fronzoni, D. D. Tommaso, and P. Decleva, J. Chem. Phys. 120, 3284 (2004).

[18] N. Böwering, T. Lischke, B. Schmidtke, N. Müller, T. Khalil, and U. Heinzmann, Phys. Rev. Lett. 86, 1187 (2001).

[19] U. Hergenhahn, E. Rennie, O. Kugeler, S. Marburger, T. Lischke, I. Powis, and G. Garcia, J. Chem. Phys. 120, 4553 (2004).

[20] G. A. Garcia, L. Nahon, M. Lebech, J.-C. Houver, D. Dowek, and I. Powis, J. Chem. Phys. 119, 8781 (2003).

[21] A. Rich, J. van House, and R. A. Hegstrom, Phys. Rev. Lett. 48, 1341 (1982).

[22] P. S. Farago, J. Phys. B 13, L567 (1980); 14, L743 (1981).

[23] R. Fandreyer, D. Thompson, and K. Blum, J. Phys. B 23, 3031 (1990).

[24] S. Mayer and J. Kessler, Phys. Rev. Lett. 74, 4803 (1995).

[25] C. Nolting, S. Mayer, and J. Kessler, J. Phys. B 30, 1997 (1997). 
[26] J. Kessler, Aust. J. Phys. 51, 645 (1998).

[27] C. Westphal, dissertation, Universität Bielefeld (1991).

[28] M. J. Frisch et al., GAUSSIAN 98, Revision A.6 (Gaussian, Inc., Pittsburgh, PA, 1998).

[29] M. Getzlaff, and G. Schönhense, J. Electron Spectrosc. Relat. Phenom. 95, 225 (1998).

[30] F. Schäfers, W. Peatman, C. Heckenkamp, A. Eyers, G. Schönhense, and U. Heinzmann, Rev. Sci. Instrum. 57, 1032 (1986).

[31] KBL 210, sensitive entrance $10 \times 2 \mathrm{~mm}$, Dr. Sjuts Optotechnik GmbH, D-37007 Göttingen, Germany.

[32] G. Martinez, M. Sancho, and F. H. Read, J. Phys. E 16, 631 (1983).

[33] I. Novak, S. C. Ng, and B. Kovac, Spectrochim. Acta, Part A 49, 1629 (1993).

[34] I. Novak and B. Kovac, J. Electron Spectrosc. Relat. Phenom. 70, 259 (1995).
[35] J. Pollmann, R. Franke, and J. Hormes, Spectrochim. Acta, Part A 53, 491 (1997).

[36] T. A. Carlson, G. E. McGuire, A. E. Jonas, K. L. Cheng, C. P. Anderson, C. C. Lu, and B. P. Pullen, in Electron Spectroscopy, edited by D. A. Shirley (North-Holland, Amsterdam, 1972), p.207

[37] R. M. White, T. A. Carlson, and D. P. Spears, J. Electron Spectrosc. Relat. Phenom. 3, 59 (1974).

[38] J. T. J. Huang and J. W. Rabalais, in Electron Spectroscopy: Theory, Techniques and Applications, edited by C. R. Brundle and A. D. Baker (Academic, London, 1978), Vol. 2, p. 197.

[39] F. A. Grimm, J. D. Allen, T. A. Carlson, M. O. Krause, D. Mehaffy, P. R. Keller, and J. W. Taylor, J. Chem. Phys. 75, 92 (1981).

[40] I. Novak, L. Klasinc, B. Kovac, and S. P. McGlynn, J. Mol. Struct. 297, 383 (1993). 